\title{
Public Affairs aus der Perspektive von Politik und Verwaltung: Nutzen und Grenzen
}

\author{
Nils Napierala und Andrea Römmele
}

\section{Inhalt}

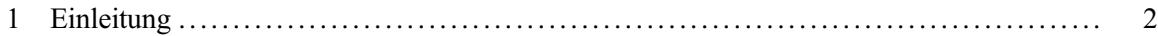

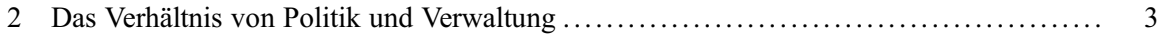

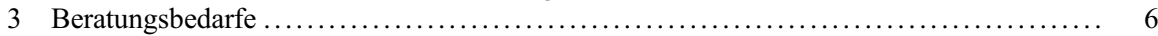

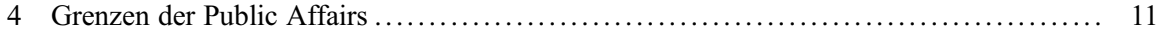

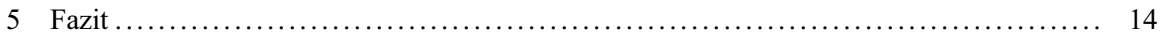

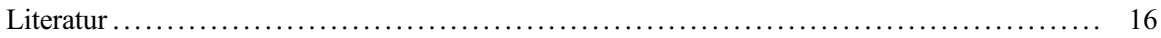

\section{Zusammenfassung}

Politik und Verwaltung treffen Entscheidungen von enormer Komplexität. Diese betreffen unterschiedlichste Akteure, berühren verschiedenste Fachgebiete, sind in ihren Konsequenzen niemals zweifelsfrei vorhersehbar, stets kontingent und erfordern ein hohes Maß an Wissen. Dieses Wissen wird nicht nur aktiv eingeholt, sondern verschiedene Akteure versuchen es strategisch im politischen Prozess zu platzieren. Sie betrachten dieses aktive Vorgehen als Voraussetzung dafür, dass ihre Interessen bei politischen Entscheidungen ausreichend berücksichtigt werden. Der vorliegende Artikel nähert sich dieser Form der interessengeleiteten Einbringung von Wissen in den politischen Prozess aus der Perspektive von Politik und Verwaltung. Nach einer Einführung in die Thematik sollen der Nutzen, aber auch die Grenzen aufgezeigt werden, die die Public Affairs für Politik und Verwaltung haben.

\section{Schlüsselwörter}

Public Affairs · Politikberatung · Interessenvertretung $\cdot$ Politikrelevantes Wissen $\cdot$ Repräsentation

N. Napierala $(\bowtie) \cdot$ A. Römmele Hertie School, Berlin, Deutschland

E-Mail: napierala@hertie-school.org; roemmele@hertie-school.org 


\section{$1 \quad$ Einleitung}

Wissen, Rat und Expertise von Akteuren aus Wirtschaft, Wissenschaft und Zivilgesellschaft in politische Entscheidungen einfließen zu lassen ist ein zutiefst demokratischer Vorgang. Solange wir politische Entscheidungsträger als Repräsentanten der Gesellschaft betrachten, müssen wir ihnen die gleichen Grenzen zugestehen, die wir auch an uns selbst beobachten. So wie auch wir uns bei unterschiedlichsten Entscheidungen Rat von außen holen, profitieren auch Politik und Verwaltung von der Unterstützung verschiedener Experten. Neben eigenen Mitarbeitern, den wissenschaftlichen Diensten, selbst initiierten Kommissionen oder den wissenschaftlichen Beiräten in den Ministerien kann dieses Wissen auch von externen Akteuren wie Kanzleien, Agenturen, Verbänden, Think Tanks, Stiftungen und wissenschaftlichen Instituten in politische Entscheidungsprozesse eingebracht werden. Formen der Interessenvertretung von Akteuren gegenüber dem politischen System werden im Folgenden als Public Affairs bezeichnet. Sie beschreiben zunächst einfach „öffentliche Angelegenheiten“. Aus Sicht eines Unternehmens beispielsweise das ,[...] Arbeitsfeld der politischen Beteiligung und öffentlichen Kommunikation an der Schnittstelle zu Politik, Gesellschaft und Medien“"(de|ge|pol 2010, S. 9). Spezifischer lassen sich als Public Affairs alle strategischen Maßnahmen von Akteuren fassen, die darauf abzielen, politische Entscheidungsprozesse zu beeinflussen. Dabei spielt es keine Rolle, ob ein Akteur aus der Wirtschaft oder der Zivilgesellschaft kommt. Gleiches gilt bei der Frage, ob er selbst mit der Politik in Kontakt tritt, oder sich von Verbänden oder Dienstleistungsanbietern vertreten lässt.

Dieses Feld wächst nicht nur, ${ }^{1}$ sondern wird auch immer ausdifferenzierter und bedeutender (Falk et al. 2010). Während die interne und/oder wissenschaftliche Beratung als notwendig anerkannt ist, steht die externe und/oder interessengeleitete Beratung immer wieder in der Kritik. Eine Bereitstellung von scheinbar ,objektivem“ Wissen wird akzeptiert und ist auch Legitimationsquelle von Politik (Head 2010), Formen der Interessenvertretung werden aber misstrauisch beäugt. Häufig wird eine Unterscheidung vorgenommen, bei der auf der einen Seite von einem beraterischen wissensgeleiteten Ansatz und auf der anderen Seite von einem beeinflussenden interessengeleiteten Ansatz ausgegangen wird. In der Praxis ist diese Grenze in der Regel jedoch nicht haltbar und auch nicht grundsätzlich normativ wünschenswert. Sowohl Information als auch Interessen sind notwendige Bestandteile politischer Entscheidungsfähigkeit. Nicht ohne Grund finden sich in vielen politischen Beratungsgremien, beispielsweise in der Hartz-, oder der Mindestlohn-Kommission, sowohl Wissenschaftler als auch Interessenvertreter (Hustedt et al. 2010, S. 16).

\footnotetext{
${ }^{1}$ Aufgrund des nicht vorhandenen Lobbyregisters in Deutschland lassen sich keine genauen Zahlen angeben. Schätzungen gehen meist von Zahlen zwischen 5000 und 6000 Interessenvertretern in Berlin aus. Das Verbänderegister des Deutschen Bundestags führt in seiner Fassung vom 08.11.2019 insgesamt 2323 Verbände auf (1980 lag diese Zahl noch unter 1000). Die Liste basiert allerdings auf Freiwilligkeit und Public-Affairs Büros von Unternehmen, Agenturen oder Einzelberater werden nicht aufgeführt. Aktivitäten in Ministerien und anderen Verwaltungsbehörden werden ebenfalls nicht angezeigt und auch auf Länderebene lassen sich keine Aussagen treffen.
} 
Die Public Affairs nehmen dabei eine doppelt beratende Funktion ein: sie beraten auf der einen Seite ihre Kunden im Umgang mit politischen Entscheidungsträgern, aber ebenso jene Entscheidungsträger, denen sie neben Fachwissen auch Interessen in aufbereiteter Form zur Verfügung stellen. Daher ist es nicht verwunderlich, dass auch Ministerien in der Gesetzgebungsphase vermehrt Public Affairs Akteure einladen (Enke und Franke 2019, S. 531). Die Notwendigkeit von externer Expertise lässt sich außerdem mit dem Wunsch nach einer qualitativen Entwicklung staatlichen Handelns sowie seiner Expansion begründen. Seit dem 19. Jahrhundert lässt sich eine Ausweitung der Staatsfunktionen beobachten, die derzeit besonders im Bereich Umweltschutz und Risikoregulierung hervortritt (Weingart 2019, S. 68). Politik greift immer tiefer in politikferne Prozesse ein und benötigt sowohl für die Entscheidungsfindung als auch die anschließende Legitimation in immer höheren Maße Spezialwissen.

Das Verhältnis zwischen dem politischen System und den Public Affairs ist dabei am besten als symbiotisch zu beschreiben. Mit Rückgriff auf Habermas (1968) hat Grundwald (2008) eine Typologisierung der Politikberatung entwickelt. Dabei unterscheidet er zwischen dem dezisionistischen, dem technokratischen und dem pragmatisch-diskursiven Modell. Während im dezisionistischen Modell die Beratung ausschließlich der Unterstützung der Entscheidungsträger dient, geht es im technokratischen Modell um vermeintliche Wahrheiten, die aus der Beratung in die Politik transferiert werden und von dieser anschließend nur noch umgesetzt werden müssen. Im Gegensatz zu diesen beiden Modellen ruft Habermas mit dem pragmatisch-diskursivem Modell Berater und Beratene zu einem kontinuierlichen Austausch auf, um mangelnde Souveränität der Beratung im dezisionistischen und mangelnde Legitimität im technokratischen Modell auszugleichen. Dann benötigen sich Politik, Verwaltung und Public Affairs gegenseitig und profitieren am Ende auch voneinander.

Die unterschiedlichen Bedarfe von Politik und Verwaltung lassen sich nicht eindeutig und trennscharf definieren. Vielmehr müssen sie sowohl von Problemstellung zu Problemstellung, als auch aus den strukturellen und funktionalen Unterschieden abgeleitet werden. Im Folgenden soll zunächst auf das Verhältnis von Politik und Verwaltung und ihre verschiedenen Funktionslogiken geblickt werden, um daraus die unterschiedlichen Nutzen und Grenzen der Public Affairs abzuleiten.

\section{Das Verhältnis von Politik und Verwaltung}

Die klassische Perspektive auf Politik und Verwaltung war lange Zeit von der Bürokratietheorie Max Webers geprägt. Demnach findet sich in der Organisation der Politik eine Trennung zwischen Werten und Fakten, die sich in einer Arbeitsteilung zwischen Politikern und Beamten widerspiegelt (1985). Die Verwaltung ist Teil der Exekutive und führt bestimmte Aufgaben im Sinne der Regierung aus. Sie soll rational handeln, um zu garantieren, dass niemand bevorzugt oder benachteiligt wird, da sämtliche Regeln für alle gleichermaßen Gültigkeit besitzen. Der Verwaltung wird dabei eine rein umsetzende Rolle zugesprochen. In dieser Vorstellung 
definiert die Politik Aufgaben und Ziele, die die Verwaltung anschließend umsetzen muss (Schedler und Eicher 2013, S. 371).

Diese Vorstellung wurde jedoch von der neueren Verwaltungsforschung aufgegeben. Man geht davon aus, dass die Aufgabenstellungen aus der Politik meist nicht so klar definiert sind, dass die Verwaltung nicht selbst immer wieder auch politische Entscheidungen treffen müsste (Schedler und Eicher 2013, S. 371). Verwaltung kann nicht mehr nur als rein ausführendes, sondern muss auch als gestaltendes Organ verstanden werden (Nicholson-Crotty 2005). Dies gilt zum einen für die Phase der Politikumsetzung. Für sie zeigte Lipsky (1980) bereits in seiner „Street level bureaucracy theory“, dass sich die Verwaltungsmitarbeiter in manchen Situationen nicht an Vorgaben halten, weil sie beispielsweise nicht spezifisch genug formuliert sind, um stets als Handlungsanweisung auszureichen. Die unklaren Zielvorgaben aus der Politik entspringen oftmals der Komplexität des politischen Systems und seiner Aufgaben, da die Mitsprache verschiedenster politischer und administrativer Akteure eine systematische Politikvorbereitung erschweren (Schedler und Eicher 2013, S. 371-372). Innerhalb dieser Interpretationsspielräume kann die Verwaltung Einfluss ausüben, indem sie beispielsweise die politischen Instrumente auswählt, mit dem Ziele der Politik erreicht werden sollen (Bogumil 2002, S. 15).

Neben Spielraum in der Phase der policy implementation wird der Verwaltung mittlerweile auch schon in der Phase des policy making eine Rolle zugeschrieben. Eine der zentralen Aufgaben der Verwaltung ist das Versorgen der Politik mit notwendigen Informationen. Meist bedeutet dies die Zusammenstellung von Informationen, zur Entscheidungsfindung der Politik, die aufgrund von Kapazitätsgrenzen nicht selbst dazu in der Lage ist. So werden im Gesetzgebungsprozess üblicherweise die fachlichen und rechtlichen Fragen an die Verwaltung gegeben, die Referentenentwürfe entwickelt, die anschließend von der Politik diskutiert werden. Hier können nicht nur eigene Ideen der Verwaltung einfließen, sondern durch Auslassungen auch Gestaltungsspielräume für die Verwaltung Eingang in Gesetze finden (Grohs 2015, S. 98). Aber auch die Identifikation von Problemen und die Erarbeitung von Alternativen können von der Verwaltung vorgenommen werden (Bogumil 2002, S. 15). Dadurch erhält die Verwaltung eine politische Aufgabe, da die Selektion davon, welche Informationen relevant sind und welche nicht, bereits zutiefst politisch ist (Schedler und Proeller 2000, S. 23). Dies ermöglicht nicht nur die Selektion von entscheidungsrelevanten Informationen, sondern auch Programmanstöße, die Ausarbeitung von Handlungsalternativen oder auch Widerstand gegen bestimmte Vorschläge (Grohs 2015, S. 97). Der hohe Einfluss, den Verwaltung in der Entscheidungsvorbereitung hat, wird häufig auf ihre Expertise zurückgeführt, die meistens derjenigen der Politik überlegen ist. Darüber erhält die Verwaltung auch empirisch nachweisbaren Einfluss in den Phasen der Problemdefinition, dem AgendaSetting und der Entscheidungsvorbereitung (Grohs 2015, S. 97) noch bevor ein Problem auf der politischen Bühne auftaucht.

In einem hochdifferenzierten, pluralistischen und korporativen Verhandlungsstaat steigt die Bedeutung der Verwaltung bei der Formulierung und Umsetzung von Politik (Bogumil 2002, S. 18) und sie ist sowohl bei der Aggregation, als auch der Artikulation gesellschaftlicher Interessen beteiligt (Lehmbruch 1987, S. 263). Ent- 
gegen dem Bild des neutralen Beamten zeigt die verwaltungswissenschaftliche Forschung, dass die Verwaltung nicht unpolitisch agiert und bereits im Gesetzgebungsprozess eine wichtige Rolle spielt sowie große Handlungsspielräume während der Programmumsetzung besitzt (Grohs 2015, S. 101). Bogumil kommt zu dem Ergebnis, dass das Bild der kooperativen Verwaltung am ehesten der Verwaltungswirklichkeit entspricht (Bogumil 2003, S. 72). Dabei kommt der Verwaltung beim policy making und bei der policy implementation die Rolle eines Partners der Politik zu (Bogumil 2003, S. 66). In Anlehnung an Peters (1987) beschreiben auch Biegelbauer et al. (2013, S. 230) die meisten Fälle von einer „funktional-pragmatischen oder freundschaftlichen Beziehung “ zwischen Politik und Verwaltung geprägt.

Die jeweiligen Besonderheiten von Politik und Verwaltung, aus denen heraus sich der jeweilige Nutzen und die Grenzen der Public Affairs ableiten lassen, soll im folgenden Unterkapitel herausgearbeitet werden.

\subsection{Die Funktionslogiken von Politik und Verwaltung}

Die enge Verknüpfung von Politik und Verwaltung darf nicht zu dem Fehlschluss führen, dass die beiden quasi als Einheit betrachtet werden können. Verwaltung und Politik überschneiden sich zwar teilweise in ihren Funktionen, übernehmen aber dennoch unterschiedliche Rollen (Schedler und Eicher 2013, S. 376). Sie folgen beide unterschiedlichen Rationalitäten die ihr Handeln bestimmen. Dies lässt nicht auf ein allzu beschränktes Weltbild auf Seiten der Entscheidungsträger schließen, sondern ist ein notwendiger Mechanismus zur Reduktion von Komplexität. Sie betrachten die Welt sowie die Informationen, ,[...] through a lens consisting of their preexisting beliefs" (Sabatier 1998, S. 109), die die Selektion von Wissen steuern und Entscheidungsfindung bei begrenzter Aufmerksamkeit und Zeit erst ermöglichen.

Ihre jeweiligen Handlungslogiken definieren eine bestimmte Form der Sinnerschließung, die wie ein Filter die Wahrnehmung der Umwelt bestimmt und zu spezifischen Wirklichkeitskonstruktionen führt (Schedler 2003, S. 538-539). Was von der Verwaltung als rationale Sachentscheidung interpretiert wird, kann von der Politik als unmöglich bewertet werden, weil beispielsweise die notwendigen politischen Mehrheiten fehlen. Solche Mehrheiten werden häufig in Aushandlungsprozessen kreiert, die wiederum für die Verwaltung irrational erscheinen können (Schedler und Proeller 2000, S. 53). Während die politische Rationalität darauf ausgerichtet ist in Verhandlungen Koalitionen zu schmieden, Menschen von Positionen zu überzeugen und relevante Stakeholder auf die eigene Seite zu ziehen, um Mehrheiten zu erlangen, ist in der Rationalität der Verwaltung die Erfüllung von Staatsaufgaben das formale Erfolgskriterium (Schedler und Eicher 2013, S. 379). Konkretisiert auf einzelne Akteure bedeutet dies, dass Politiker ein Interesse daran haben wiedergewählt zu werden, um ihre politischen Ziele erfüllen zu können und ihre Rationalität dadurch stark vom Wählerwillen beeinflusst wird. Akteure aus der Verwaltung versuchen hingegen die Umsetzung der ihnen übertragenen Aufgaben möglichst gut zu erfüllen. Pierre und Peters haben ebendies in empirischen Unter- 
suchungen bestätigen können. Demnach wollen Bürokraten ihre Arbeit gut erledigen, um einen Beitrag für das Gemeinwohl zu leisten (Pierre und Peters 2017) und sind dafür auf Legalität, Effektivität und Effizienz als Rationalitätskriterien angewiesen.

Die verschiedenen Handlungslogiken lassen sich aber nicht nur aus den unterschiedlichen Rationalitäten ableiten, sondern ebenfalls aus strukturellen Unterschieden zwischen Politik und Verwaltung. Dabei kommen insbesondere zwei Faktoren zum Tragen: Unterschiedliche Spezialisierungsgrade sowie unterschiedliche Zeithorizonte. Zwar gibt es Fachpolitiker, die sich auf bestimmte Bereiche spezialisiert haben, oder bereits vor ihrer politischen Tätigkeit Spezialisten waren, dennoch besteht für sie die Anforderung als Generalisten zu fungieren, da sie potenziell zu unterschiedlichsten Themen Stellung nehmen und Entscheidungen treffen müssen sowie viel Kapazität für politikspezifische Aufgaben wie Wahlkreisarbeit, Wahlkämpfe, öffentliche Auftritte, Parteiarbeit etc. aufwenden müssen. Die Verwaltung ist hingegen von der Spezialisierung und hoher Expertise geprägt. Dies hängt auch mit den unterschiedlichen Zeithorizonten zusammen. Politik ist in demokratischen Verhältnissen immer zeitlich begrenzt und findet im Rhythmus der Wahlzyklen statt. Demgegenüber ist die Verwaltung deutlich konstanter. Zwar existiert in Deutschland eine „politisierte Ministerialverwaltung“ (Bogumil et al. 2012), bei der Spitzenbeamte wie Staatssekretäre und Abteilungsleiter häufig ihre Positionen verlieren, wenn es an der Spitze ihres Ressorts zu Veränderungen kommt, aber nichtsdestotrotz agiert ein enormer Anteil der Verwaltung unabhängig von Wahlkämpfen (Bogumil et al. 2012).

Wie im Folgenden gezeigt werden soll, führt dies zu Beratungsbedarfen auf Seiten von Politik und Verwaltung, die von Public Affairs unterschiedlich bearbeitet werden müssen.

\section{Beratungsbedarfe}

Wie gezeigt, sind Politik und Verwaltung zwar nicht gleichermaßen, aber doch beide im politischen Entscheidungsprozess beteiligt. Politische Entscheidungen erfordern stets eine Selektion aus möglichen Alternativen. Es existiert ein Überangebot an verfügbarem Wissen, bei dem entschieden werden muss, welches Wissen für die jeweilige Entscheidung relevant ist. Der Wandel hin zur Wissensgesellschaft (Stehr 1994) bedeutet auch für die Politik und die Verwaltung, dass sie Entscheidungen auf der Grundlage von hochkomplexem, aber gleichzeitig unvollständigem Wissen treffen müssen. Sie handeln stets unter Einschränkungen und begrenzt rational (Simon 1959). Übersteigen die Informationsbeschaffungskosten den erwarteten Nutzen oder sind schlicht nicht aufzubringen, wird eine Entscheidung unter Unsicherheit getroffen (Simon 1959, S. 269-270). Bei der enormen Komplexität der Sachverhalte, der typischen Kontingenz politischer Entscheidungen und dem Zeitdruck unter dem sie getroffen werden müssen, ist diese Unsicherheit die Regel. Mehr Wissen oder Information führen zwar nicht automatisch zu weniger Kontingenz im Entscheidungsprozess, sind aber für die Rückversicherung der eigenen Entscheidungswahl und insbesondere die Legitimierung von Entscheidungen elementar. 
Für die qualitative und quantitative Erweiterung der Regierungstätigkeiten unter demokratischen Vorzeichen wird neben Spezialwissen aber auch eine Repräsentation der in der Gesellschaft vorhandenen Interessen benötigt. Grundsätzlich sind diese beiden Aspekte in den Institutionen und Verfahren staatlicher Willensbildung integriert. Daher kann keine unbedingte Notwendigkeit für die externe Beratung abgeleitet werden (Graser 2019, S. 44). Jedoch kann durch eine Steigerung dieser beiden Aspekte durch Public Affairs die Qualität politischer Entscheidungen in dem Sinne erhöht werden, dass mehr Informationen verfügbar sind und mehr Interessen angehört werden. Public Affairs übernehmen dabei eine Übersetzungsleistung (Chahoud 2010, S. 35) zwischen dem politischen System und dem entsprechenden anderen. Public Affairs sind ein Teil des Austausches zwischen Gesellschaft und Politik, der jenseits von Wahlen stattfindet. Sie repräsentieren die Interessen ihrer Auftraggeber, oder initiieren einen Dialog zwischen Politik und ihnen. Dass sie dazu auch notwendig sind, zeigt eine Befragung von Spitzenpolitikern aus Bund und Ländern. Diese gaben an, dass es bei Kontakten, die direkt aus der Wirtschaft kommen, häufig an Verständnis für Agenden und Durchsetzungschancen in der Politik fehlt. $78 \%$ der Befragten gaben an, dass bei Managern das Bewusstsein für politische Herausforderungen und Grenzen fehle (Oltmanns und Seidenglanz 2020). Dieses Bewusstsein mitzubringen muss Kernbestandteil der Public Affairs sein.

\subsection{Beratungsbedarf der Verwaltung}

Die Politik kann ihre Aufgaben nicht wahrnehmen, ohne dass die Verwaltung sie mit ihrer eigenen Expertise dabei unterstützt, Programme zu formulieren, den Dialog mit der Gesellschaft zu führen und beschlossene Programme umzusetzen. Ungefähr drei Viertel der Gesetzesinitiativen, die in den Bundestag eingebracht werden, stammen von der Bundesregierung und damit in der Regel aus Referaten der verschiedenen Ministerien (Rudzio 2015, S. 243). Bach und Wegrich definieren dabei eine hohe fachliche Unabhängigkeit der Verwaltung gegenüber der Politik als zentral, um diese „politikunterstützende Funktion“ auszuführen (Bach und Wegrich 2019, S. 65). Wie oben bereits beschrieben, sind es besonders die fachlichen und juristischen Fragen, die innerhalb des Gesetzgebungsprozesses von der Verwaltung bearbeitet werden. Gerade in sich schnell ändernden Politikbereichen benötigt die Verwaltung externe Expertise bei der Politikformulierung (Ledermann 2014, S. 460). Dabei verlässt sich die Verwaltung in erster Linie auf klassische Unternehmensberater, aber auch Wissenschaftler, Stiftungen und Verbände (Kozica et al. 2013, S. 306). Dieser Trend verdrängt seit den 1990er-Jahren zunehmend die verwaltungsinternen Beratungsinstitutionen und kommerzielle Dienstleister gewinnen an Bedeutung (Raffel 2006). ${ }^{2}$

\footnotetext{
${ }^{2}$ In einer Antwort auf eine Kleine Anfrage teilte das Bundesverwaltungsamt Ende 2019 in einer Drucksache des Bundestags (19/15632) mit, dass es weder personell noch materiell dafür ausgestattet sei, den gesamten Bedarf der Bundesverwaltung an Organisationsberatung abzudecken. Dies sei auch nicht die Aufgabe des Amtes, das aber im Rahmen seiner Kapazitäten dazu beitrage, den Beratungsbedarf der Bundesverwaltung im Sinne eines ,gesunden“ Mixes durch interne und externe Beratung zu decken.
} 
Stöbe-Blossey (2004) macht in der Verwaltung Hindernisse aus, die eine Umsetzung von externen, wissenschaftlichen Beratungsergebnissen erschweren. Die der Verwaltung eigene Rationalität führe dazu, dass Wissenschaft häufig Ergebnisse produziert, die von der Verwaltung nicht umgesetzt werden können. Zusätzlich gäbe es aber eine den Verwaltungen immanente Eigenständigkeit, die Umsetzungsprozesse erschwere (Stöbe-Blossey 2004, S. 99). Mit der Anpassung an diese Gegebenheiten steht und fällt der Nutzen der Public Affairs für die Verwaltung.

Um die Wirkungen ihrer Entscheidungen möglichst gut abschätzen zu können, ist es für die Verwaltung außerdem notwendig nicht nur Spezialwissen, sondern auch Interessen von externen Akteuren einzuholen. Je komplexer politische Entscheidungen werden und je tiefer Regulierungen in bestimmte Bereiche eingreifen, umso schwieriger wird es, daraus resultierende, mögliche Folgeprobleme zu antizipieren. Dies gelingt besser, wenn man eine Entscheidung aus einer bestimmten Interessenlage heraus bewertet, wie es die Public Affairs tun. Sie spielen die Auswirkungen von Entscheidungen auf die sie betreffenden Bereiche durch, geben ihre Ergebnisse in den Gesetzgebungsprozess zurück und übernehmen dabei eine Aufgabe, die die Verwaltung aufgrund begrenzter Kapazitäten und unterschiedlicher Perspektiven unmöglich erfüllen kann. Die Expertise der Public Affairs entspringt zu großen Teilen aus der Betroffenheit der Vertretenen. Eine Funktion, die umso wichtiger wird, als immer mehr Entscheidungen auf einer supranationalen Ebene getroffen werden, auf der eine organisierte und konzentrierte Vertretung von Interessen sowohl notwendiger, als auch schwieriger wird, da die Anzahl der in unterschiedlichem Maße Betroffenen enorm ansteigt.

Ist ein Gesetz beschlossen und befindet sich in der Implementierungsphase, besteht häufig auch noch Potenzial zu Veränderungen, da Politik Regelungen nicht immer so kleinteilig formulieren kann, dass die Administration keinen Spielraum mehr hat. Daumann bezeichnet daher in der Implementierungsphase die Vertreter der öffentlichen Verwaltung als zentrale Handlungsträger (Daumann 1999, S. 216). Bei der Ausgestaltung dieses Spielraums kann externe Expertise nützlich und Interessenvermittlung erfolgreich sein. Ein eindrückliches, wie auch bezeichnendes Beispiel dafür war das Engagement der Kanzlei Linklaters, die im Juli 2009 an einem Wochenende den Entwurf für das „Gesetz zur Änderung des Kreditwesengesetzes“ anfertigte. Dies warf allerdings zum einen die Frage auf, ob das Parlament über die notwendige Expertise verfüge, um Entscheidungen eigenverantwortlich zu treffen und zum anderen, ob die Ministeriumsverwaltung unter den vorliegenden Bedingungen nicht in der Lage sei, selbst einen Entwurf vorzulegen (Kloepfer 2011).

\subsection{Beratungsbedarf der Politik}

Die Einbeziehung von Spezialwissen sowie Interessen ist für die Entscheidungsfindung der Politik ebenfalls zentral. Anders als die Verwaltung, kann sich die Politik aber bei fachlichen und/oder juristischen Fragen stets auf die Verwaltung berufen. Die Politik kann sich aber nicht allein am vorhandenen, vermeintlich objektiven Fachwissen orientieren. Selbst die Naturwissenschaften können ,[...] trotz ihrer 
scheinbar überlegenen Methodik und Modellbildung - die Rationalität politischer Entscheidungen nicht gewährleisten [...]“" (Kusche 2008, S. 12). Außerdem liefert sie Antworten, die zwar das vermeintlich optimale, nicht aber unbedingt das ökonomisch und vor allem politisch sowie gesellschaftlich Mögliche aufzeigen. Daher sind auch andere Formen von Wissen bei der Ausgestaltung politischer Maßnahmen notwendig.

Die Verhältnisse unter denen Politik agiert haben sich verändert und werden besonders von einem stetig zunehmenden Kommunikationsdruck geprägt. Die Rechtfertigung politischer Entscheidungen geschieht in Demokratien über die Kommunikation der Informationsabwägungen, die zu ihr geführt haben. Der Verweis auf „fachliche Notwendigkeiten“ reicht nicht aus, um den Anforderungen an politische Kommunikation gerecht zu werden (Bach und Wegrich 2019, S. 70). Politische Akteure wollen ihre Entscheidungen nicht nur formal begründen, sondern streben immer auch nach Machtgewinn oder -erhalt. Hier liefern Public Affairs einen Nutzen für sie, den eine fachbezogene Beratung aus Wissenschaft und/oder Verwaltung aufgrund unterschiedlicher Rationalitäten nicht liefern kann: Ergebnisse, die von den Public Affairs für die Politik aufbereitet werden, sind an politische Prozesse und die Logik der Macht angepasst. Politik und Public Affairs operieren in ähnlichen Rationalitäten und bewerten Wissen unter dem Maßstab des politischen Nutzens, dem Gewinn oder dem Erhalt von Macht. ${ }^{3}$ Public Affairs kennen die politischen Prozesse, können die politische Relevanz von Wissen einschätzen und sich daran anpassen. Sie können daher Bedarfe der Politik bedienen, die die Wissenschaft oder auch die Verwaltung nicht bedienen können. Beispielsweise haben Politik und Wissenschaft völlig unterschiedliche Zeitrationalitäten (Vorderstraße 2014). Taucht ein Problem auf der Agenda auf, kann die Politik nicht darauf warten, bis dieses wissenschaftlich bearbeitet worden ist. Die widersprüchlichen, sich je nach Kontext und Zeitpunkt ändernden Anforderungen an politisch relevantes Wissen (Straßheim 2013) und kulturelle sowie kommunikative Hürden zwischen Wissenschaft, Verwaltung und Politik erschweren die Integration von Ergebnissen noch weiter. Public

\footnotetext{
${ }^{3} \mathrm{Im}$ Gegensatz dazu, bewertet die Verwaltung, wie oben beschrieben, Wissen danach, ob es bei der Steigerung von Effizienz und Effektivität helfen kann. Wissenschaft wiederum bewertet Wissen anhand der Kategorie Wahrheit. Sie funktioniert nach bestimmten Verfahren und Praktiken, die dem wissenschaftlichen Wissen eine höhere Autorität als anderem Wissen verleihen (Knoblauch 2010, S. 238). Wissenschaftliche Beweise sind die ,[...] dominant language of legitimation and persuasion in today's liberal socities“ (Goodwin et al. 2001, S. 15). Dies könnte auch eine Erklärung dafür sein, warum die „Verwissenschaftlichung verbandlicher Expertise“ (Nullmeier 2013, S. 35) zunimmt. Die Verbandsexpertise erhält durch die Aufbereitung in Form von Studien eine „,wissenschaftliche Überformung“ und dadurch eine „doppelte Legitimität“ (Nullmeier 2013, S. 35). Eine Konvergenz von Politik und Wissenschaft lässt sich auch bei der Annäherung der Leistungsspektren von Agenturen an den wissenschaftlichen Output von Think-Tanks (Römmele und Schober 2013), die ihre Leistungen vor allem in Form von Studien und anderen Expertisen produzieren (Lentsch und Weingart 2009), sowie an der zunehmenden politisch-ideologisch geprägten Ausrichtung der Denkfabriken (Speth 2004) feststellen. Die Unterscheidung zwischen Think-Tanks und Public Affairs wird daher auch von verschiedenen Autoren in Zweifel gezogen (Siefken 2010; Leif und Speth 2006).
} 
Affairs bieten hier Beratung, die unter kompatiblen Rationalitäten arbeitet, da beide auf die Erreichung politischer Ziele ausgerichtet sind. Andernfalls würden Interessenvertreter, die ähnliche politische Ziele verfolgen, nicht häufig erste Ansprechpartner für Abgeordnete sein (Buzogány und Kropp 2013, S. 177).

Public Affairs erhoffen sich durch Zugriff auf die Politik Vorteile bei der Verwirklichung ihrer Interessen, während Politik die Einbeziehung nur zulässt, wenn Hoffnung auf mögliche Vorteile im Parteienwettbewerb besteht. Da kann eine wissenschaftliche Studie irrelevant, der Austausch mit einem Interessenvertreter aber sehr wichtig werden. Darin resultiert auch, dass Beratungsleistungen, die Parteien selbst vertraglich anfordern, in erster Linie aus den Bereichen Kommunikation und Wahlkampf entspringen (Falk und Römmele 2009; Jun 2019) und eben nicht zu einer besseren Politik, sehr wohl aber besseren Chancen im politischen Wettstreit führen. Sie müssen ihre Ideen so mit dem politischen Publikum verknüpfen, dass sie einen Vorteil auf „einem Markt des Wissens“ (Nullmeier und Rüb 1993, S. 53), der nicht davon bestimmt ist, welches Wissen vermeintlich „korrekter" oder „wahrer“ ist, sondern davon, ob es gelingt, ,[...] durch eine spezifische Aufbereitung und Konstruktion des Wissens" andere des Wissens glauben zu machen (Rüb 2006, S. 346). Elementar ist es dabei, dass die politischen Ziele der Public Affairs und der angesprochenen politischen Akteure miteinander kompatibel sind, da ansonsten der Nutzen der Public Affairs für die Politik verpufft. Dabei profitieren Beratungsprodukte von ihrem Dasein als „Grenzobjekte“. Korinek und Veit beschreiben sie als Objekte, bei denen sich Experten und Entscheider nicht auf eine gemeinsame Definition einigen müssen „Sie können ausgehend von ihren jeweiligen Problemdefinitionen und Lösungskonzepten an die Objekte anschließen, diese konkretisieren und schließlich nach ihren eigenen Handlungsprämissen verwenden" (2013, S. 271). So wird das strategische Framing eines Problems zentraler Erfolgsfaktor für die Public Affairs.

Um auf dem Markt des Wissens zu bestehen, müssen politische Akteure ihre politische Umwelt kennen. Sie müssen ein Gespür für politische Entwicklungen und Stimmungen haben, um die Widerstände und Chancen einschätzen zu können, die ihre eigenen Ziele behindern oder sie unterstützen können. In hochgradig differenzierten Gesellschaften sind die vielfältigen Interessen der Gesellschaft aber nicht automatisch im Parlament abgebildet. Public Affairs leisten hier einen Beitrag, da sie frei von den für Parteien gültigen Restriktionen sind und den sozialen Pluralismus feinmaschiger wiedergeben (van Schendelen 2014, S. 670). Dabei nehmen insbesondere Verbände eine wichtige Rolle ein, die als organisierte Interessenvertretungen eine Vorsortierung und Filterung gesellschaftlicher Interessen vornehmen (Wagschal 2019, S. 57). Interessenvertretung kann eine Möglichkeit der Rückbindung der Politik an bestimmte gesellschaftliche Gruppen sein. Gerade auf EU-Ebene wird die Bedeutung des Austausches zwischen Interessenvertreten und Politik deutlich. Stellt der Austausch für Abgeordnete doch eine Möglichkeit dar, enger an den vielfältigen Interessen der gut 513 Millionen EU-Bürger zu sein (Wonka 2005, S. 171-172).

Politik und Verwaltung benötigen möglichst unumstrittenes, nach wissenschaftlichen Methoden und Kriterien produziertes Wissen, um Entscheidungen sowohl zu 
treffen, aber auch um sie zu legitimieren. Um wissenschaftliche Expertise in handlungsrelevantes Wissen zu transferieren, müssen Politik und Verwaltung selbst erhebliche Ressourcen investieren. Beispielsweise durch die Anpassung an eigene Problemdefinitionen und Lösungsalternativen oder eine möglichst schillernde Aufbereitung, von denen sie sich eine höhere Überzeugungskraft versprechen. Public Affairs können, bei entsprechenden gemeinsamen Interessen, große Teile dieser Arbeit übernehmen und das Wissen für Politik und Verwaltung bereits so weit aufbereiten, dass es von diesen direkt für die eigene Arbeit angewandt werden kann. Es ist die Verbindung der verschiedenen Formen von Wissen und Rationalitäten, die den Public Affairs eine besondere Bedeutung für Politik und Verwaltung geben. Die Motivation für solches Verhalten ist auf Seiten der Public Affairs extrem hoch. Je besser sie sich an die jeweiligen Rationalitäten von Politik und Verwaltung anpassen, umso anwendbarer und sinnvoller werden ihre Leistungen für diese und ums höher werden die Chancen der Public Affairs ihre Ziele zu erreichen. Dennoch gibt es auch unüberwindbare Grenzen zwischen den Public Affairs, der Politik und der Verwaltung.

\section{$4 \quad$ Grenzen der Public Affairs}

Egal, wie nützlich der Input der Public Affairs ist, die politische Entscheidungsgewalt muss immer bei Institutionen liegen und in Verfahren vollzogen werden, um demokratische Legitimation zu garantieren. Grundsätzlich ist es so, dass der zugrunde liegende Input die Legitimität einer Entscheidung nicht beeinflusst, solange die öffentliche Gewalt die Entscheidung unabhängig trifft (Burkhardt 2008, S. 189-190). Durch mangelnde Transparenz kann aber eben diese Unabhängigkeit nicht zweifelsfrei gewährleistet werden, was die Legitimation durch Verfahren (Luhmann 1989) zunichtemacht. Dies ist der Fall, wenn nicht mehr zweifelsfrei nachvollzogen werden kann, ob die öffentliche Gewalt Entscheidungen tatsächlich getroffen oder lediglich bestätigt hat. Dies muss zu einem gewissen Grad ausgehalten werden, da demokratische Entscheidungen von der Politik nur getroffen werden können, wenn es eine bottom-up Informationsübertragung aus der Gesellschaft gibt und Interessen an Entscheider vermittelt werden. Selbstverständlich ist es aber nicht Aufgabe von Politik und Verwaltung, sämtliche Interessen einfach in Gesetzesform zu gießen, sondern es geht darum, Interessen gegeneinander auszugleichen und Kompromisse zu finden. Dabei stoßen die Public Affairs an ihre Grenzen. Sie sind selbst auf die Umsetzung ihrer Interessen aus. Kompromiss und Ausgleich sind für sie strategisch anwendbare Werkzeuge, um diese Ziele zu erreichen, aber keine ihnen immanenten Eigenschaften. Public Affairs sind für Politik und Verwaltung bei Interessenausgleich und Kompromissfindung zwar notwendig, können sie aber nur bedingt unterstützen, beziehungsweise durch eine unverhältnismäßig starke Repräsentation sogar behindern.

Aufgrund unterschiedlicher Ressourcenverteilung ist eine gleichberechtigte Repräsentation der Interessen nicht gegeben und der Pluralismus divergierender Positionen spiegelt sich in den Public Affairs nicht wider. Der Blick nach Brüssel zeigt 
beispielsweise, dass die Mehrzahl von Public Affairs-Akteuren, -Maßnahmen und -Netzwerken aus Deutschland, Frankreich und Großbritannien stammen. Auch auf nationaler Ebene lässt sich eine Vielzahl von sozialen Gruppen identifizieren, denen keine Interessenvertretung zur Seite steht. Es gibt zwar auch öffentliche Akteure, die Public Affairs Maßnahmen betreiben (bspw. Industrie- und Handelskammern oder Zusammenschlüsse von Städten oder Hochschulen (Baumgartner et al. 2009)), aber die meisten Aktivitäten gehen von gesellschaftlichen, und hier mit großem Abstand unternehmerischen Akteuren, aus (Bernhagen 2019, S. 251). Ressourcen, und damit die Möglichkeiten, Interessen an die Politik zu artikulieren, sind unter den verschiedenen Gruppen und Akteuren ungleich verteilt. Die derzeitigen Regulierungs- und Kontrollmechanismen sind zu schwach, um dem entgegenzuwirken. Aus den verfügbaren Ressourcen eines Akteurs lässt sich allerdings nicht automatisch die Relevanz des inhaltlichen Einflusses ableiten (Schmedes 2010, S. 26). Es ist zwar eine notwendige Voraussetzung, aber keine Garantie für die erfolgreiche Durchsetzung eigener Interessen. Auch extrem hohe Ressourcen versprechen nicht automatisch Erfolg. Umgekehrt bedeutet dies, dass selbst bei ausgeglichenen Ressourcen nicht automatisch eine gleichberechtigte Repräsentation erfolgt. Es gibt nämlich auch eine Ungleichbehandlung durch Politik und Verwaltung selbst.

Damit Public Affairs für politische Entscheidungsträger überhaupt interessant werden, müssen diese zunächst zeigen, dass sie einen Informationsvorsprung haben (Ainsworth 1993). Dieser allein reicht jedoch nicht aus, da der Informationsempfänger jenes Wissen bevorzugt, das den eigenen ideologischen Vorstellungen entspricht, für ihn also politisch nutzbar ist. Denn „Wenn ein Beratungsadressat bereits von etwas überzeugt ist, sei das ein Sachverhalt oder die Richtigkeit einer bestimmten Entscheidung, dann fragt er nicht mehr nach Information und Rat, sondern nur noch nach Unterstützung" (Mayntz 2006, S. 21). Politik und Verwaltung wählen Wissen nach ihnen eigenen Selektionskriterien aus, die sich aus den ihnen immanenten Logiken ergeben. Die Pluralisierung des Wissensangebots eröffnet hier ein anwachsendes Arsenal kompatibler Wissensformen, aus dem man sich bedienen kann. Kriterien wie Objektivität verlieren dann an Bedeutung, aber der Anwendungsbezug in Bezug auf Machterhalt und politische Entscheidungsgewalt sowie effiziente und effektive Erfüllung von Staatsaufgaben wird umso höher. Wissen wird im Rahmen der jeweiligen Rationalität von den Akteuren instrumentell und innerhalb einer Machtlogik bewertet. Damit ist auch klar, dass sich politische Entscheider wenig Illusionen darübermachen, dass Public Affairs immer mit bestimmten Interessen verbunden sind. In der bereits weiter oben erwähnten Befragung von Spitzenpolitikern stellte sich aber heraus, dass lediglich $15 \%$ der Befragten Wirtschaftsvertreter als transparent und offen einschätzen (Oltmanns und Seidenglanz 2020). Solange also das Bild des verdeckten Lobbyismus bestehen bleibt, ist eine vertrauensvolle Zusammenarbeit zwischen Politik und Verwaltung auf der einen, und den Public Affairs auf der anderen Seite deutlich erschwert. 


\subsection{Grenzen der Politik für die Public Affairs}

Externes Wissen stellt für Politik nicht nur einen Nutzen, sondern auch ein Risiko dar. Es kann die eigene Definitionsmacht schwächen, wenn es den eigenen Vorstellungen entgegenläuft. Weiteres Wissen macht potenziell die Kontingenz politischer Entscheidungen deutlich, deren Existenz man zur Durchsetzung eigener Ziele aber versucht zu relativieren. $\mathrm{Zu}$ jeder politischen Entscheidung existieren Alternativen, deren Existenz oder Plausibilität weder vor dem politischen Publikum, noch vor sich selbst endgültig negiert werden kann. ,[...] alternativlose Entscheidungen, denen zufolge Expertise und Wissen das Handeln von Politik und Verwaltung technokratisch programmieren, sind in einer Demokratie jedoch nicht denkbar" (Kropp und Kuhlmann 2013, S. 8). Die Selektivität des selbst gewählten Wissens durch die Einbeziehung weiteren Wissens zu reduzieren, führt dazu, dass politische Entscheidungen an Orientierung verlieren, weil Kontingenz erfahrbarer wird (Kusche 2008, S. 35). Informationen und Wissen, die der eigenen Handlungswahl entgegenlaufen, machen die Entscheidung sowohl für einen selbst, als auch Dritte unsicherer und erschweren potenziell politisches Entscheiden. Die Auswahl des heranzuziehenden Wissens ist daher ein eigenes politisches Steuerungsproblem (Weingart und Lentsch 2008, S. 37).

Durch die Auswahl der einzubeziehenden externen Wissensträger wird oftmals keine neue Sichtweise erschlossen, aber die eigene Überzeugungskraft, Entscheidungssicherheit und Legitimität erhöht. Auf diese Art und Weise ausgewähltes externes Wissen bietet eine Aufarbeitung des Wissens, die an die eigenen ideologischen Vorstellungen angepasst ist. Politik hat dann ein Interesse an externer Beratung, wenn sie zur eigenen Zielerreichung genutzt werden kann. Wissen, das beispielsweise erst in der nächsten Legislaturperiode politisch nutzbar ist, ist für aktuelle Mandatsträger uninteressant. In so einem Fall muss eine Lösung mitgeliefert werden, wie die entsprechenden Akteure von den Maßnahmen profitieren können, auch wenn sich die Effekte möglicherweise erst deutlich später einstellen. Stehen die von den Public Affairs vertretenen Ziele denen der Politik entgegen, und gelingt es ihnen nicht, für die entsprechenden Akteure politikrelevantes Wissen anzubieten, wird es zu keinem fruchtbaren Austausch kommen. Dadurch wird eine technokratische Auslagerung von politischen Entscheidungen an Experten verhindert und sichergestellt, dass die Entscheidung weiterhin politisch getroffen wird.

\subsection{Grenzen der Verwaltung für die Public Affairs}

Public Affairs werden für die Verwaltung erst interessant, wenn sie ihnen dabei helfen, die ihnen übertragenen Staatsaufgaben zu erfüllen. Wie bereits oben angeführt sind für die Verwaltung Effektivität und Effizienz sowie Legalität die entscheidenden Rationalitätskriterien. Diese stehen zwar in keinem hierarchischen Verhältnis (Bogumil und Jann 2009), aber angebotenes Wissen muss der Verwaltung einen Vorteil bei einem dieser Kriterien versprechen, um von ihr überhaupt ange- 
nommen zu werden. Angebote, die beispielsweise auf eine politische Rationalität abzielen, sind für die Verwaltung weitgehend irrelevant.

Die Verwaltung ist für die Public Affairs unter anderem deswegen wichtiger Ansprechpartner, weil Probleme hier (noch) nicht politisiert sind. Dementsprechend sind auch politische Argumente von geringerem Interesse. Positionen stehen noch nicht fest, Haltungen sind noch nicht gefunden und Expertise sowie Effektivität und Effizienz sind die zentralen Rationalitätskriterien. Auch die öffentlichkeitswirksame Gestaltung eines Themas bietet für die Verwaltung beispielsweise keinen Vorteil, da sie relativ unabhängig von Wahlzyklen agiert und keinen Bedarf daran hat, das politische Publikum für sich zu mobilisieren. Kurzfristige Maßnahmen, die für Politik interessant sein können, um beispielsweise Wähler zu mobilisieren, können für die Verwaltung daher tendenziell weniger interessant sein, als nachhaltige Lösungen, von denen sie langfristig profitiert. Aufgrund der hohen Expertise der Verwaltung stellen sich außerdem höhere Anforderungen an die fachliche Expertise der Public Affairs und es wird schwieriger die entsprechenden Akteure vom eigenen Wissensvorsprung zu überzeugen.

Die größeren Rationalitätsunterschiede zwischen Verwaltung und den Public Affairs erhöhen den Aufwand zur gegenseitigen Annäherung. So diagnostizierte der damalige Bundesminister für Finanzen Wolfgang Schäuble in einem Gastbeitrag für das Handelsblatt 2014: „Wichtig wäre ein besseres Gespür für die Logik und Zwänge der jeweils anderen Seite. Dies kann man am ehesten erreichen über einen zeitweisen Personalaustausch für den wechselseitigen Blick in die verschiedenen Welten“ (Schäuble 2014, zit. n. Schörner 2017, S. 110). Damit plädierte er für einen verstärkten Austausch und machte damit auf das Problem der verschiedenen Rationalitäten aufmerksam. Gleichzeitig machte er aber auch deutlich, dass diese zwar Hindernisse darstellen, aber nicht unüberwindbar sind.

\section{$5 \quad$ Fazit}

Im verstrickten Geflecht aus Gesellschaft, Politik und Wirtschaft nehmen die Public Affairs sowohl eine beeinflussende, aber auch eine wissens- und interessenvermittelnde Rolle ein, die nicht voneinander getrennt denkbar sind. Die Kernaufgabe von Public Affairs besteht in der Aggregierung von Interessen und Informationen und deren Aufbereitung in unterschiedlichen Wissensformen. Diese Rolle wird dabei häufig auf eine in Hinterzimmern stattfindende Beeinflussung reduziert, die der Realität zwar nicht gerecht wird, aber auch nicht aus der Luft gegriffen ist. Es kommt immer wieder zu Fällen, in denen Public-Affairs-Berater zu Methoden greifen, die mit den Grundsätzen eines demokratischen Systems nicht vereinbar sind. ${ }^{4}$ Grundsätzlich gilt aber auch unter Public-Affairs-Praktikern, dass sich langfristige Erfolge nur dann erzielen lassen, wenn man sich für verschiedene Ansprech-

\footnotetext{
${ }^{4}$ Eine kritische Auseinandersetzung mit unlauteren Methoden der Public Affairs findet sich beispielsweise bei Leif und Speth (2006); Leif (2010) oder bei NGOs wie LobbyControl.
} 
partner langfristig als glaubwürdige Quelle etabliert. Von Alemann und Eckert (2006) haben daher eine Differenzierung in eine „weiße“, eine „schwarze“ sowie eine dazwischenliegende ,graue“ Politikberatung vorgenommen, die es erlaubt sich den realen Verhältnissen exakter anzunähern. Es macht ebenso wenig Sinn die Public Affairs grundsätzlich als undemokratisch zu bezeichnen, wie es nicht zielführend ist, die schwarzen Schafe zu ignorieren.

Bewegen sich Public Affairs im Rahmen der vorhandenen gesetzlichen Regelungen, dann ist ihr Erfolg letzten Endes auch hochgradig kontextabhängig. Erfolgreiche Interessenvermittlung kann nur stattfinden, wenn es gelingt politikrelevantes Wissen zu generieren, entsprechend aufzubereiten und anschließend an die richtigen Personen zu transferieren. Die richtigen Ansprechpartner zu identifizieren ist eine der Hauptaufgaben der Public Affairs. Es gibt sowohl länder-, als auch themenspezifisch große Unterschiede, wie viel Einfluss welche Akteure aus Politik und Verwaltung auf die Politikgestaltung nehmen. So stellten Biegelbauer und Kollegen in einer international vergleichenden Studie des Einflusses von Politik und Verwaltung in der Regulation von Xenotransplantation fest, dass sich „Beamte [...] nicht darauf beschränkt [haben], die Entscheidungsfindung durch Politiker lediglich vorzubereiten. Umgekehrt haben sich Politiker nicht darauf beschränkt, lediglich auf Werten basierende Entscheidungen zu treffen“" (Biegelbauer et al. 2013, S. 236). Es besteht für die Public Affairs also eine beständige Anpassungsanforderung, die sich immer wieder verändert und eine kontinuierliche Beobachtung der eigenen Umwelt sowie ständige Anpassungen der Strategie notwendig machen. Andernfalls wiegen für Politik und Verwaltung die Grenzen der Public Affairs schwerer als ihr Nutzen.

Entscheidungsträger sind sich bewusst, dass Unternehmen und Organisationen durch Public Affairs durchaus eigene Interesse verfolgen und nicht allein auf eine reine, neutrale und interessenlose Informationsvermittlung aus sind. Es ist für sie essenziell eine Unterscheidung vornehmen zu können, mit welchen Methoden Wissen hergestellt worden ist und dies in ihre eigene Bewertung mit einfließen zu lassen. Dementsprechend können sie ihnen auch begegnen. Public Affairs können von ihnen als Mittel zur Kontingenzreduktion genutzt werden und auch wenn es ihnen nicht gelingt Alternativen völlig auszuschließen, dienen sie der Selbstvergewisserung und damit auch der Reduktion von Unsicherheit.

Public Affairs bergen für den politischen Prozess sowohl Nutzen, als auch Risiken. Sie verringern bestimmte Werte wie Transparenz, erhöhen dafür aber andere. Bringen Externe beispielsweise Wissen ein, das politische Entscheidungen verbessert, erhöht sich die Output-Legitimität von Politik (Widmer 2009). Dabei scheint insbesondere die Schärfung der Aufmerksamkeit für mögliche Konsequenzen geplanter Policy-Maßnahmen (bspw. in der Umwelt- oder Wirtschaftspolitik) einer der zentralen Nutzen der Public Affairs für Politik und Verwaltung zu sein (Bernhagen 2012), weil hier politische Entscheidungen in Feldern zentral werden, die selbst nicht zum politischen System gehören. Nichtsdestotrotz führt gerade die informelle Einbeziehung von Interessenvertretern zu einer Reduktion der Input-Legitimität. Es ist eine Abwägungsentscheidung, die auch in anderen Zusammenhängen zu 
treffen ist. Gäbe es beispielsweise eine maximale Transparenz bei sämtlichen parlamentarischen Abläufen, würde dies die Möglichkeit von Entscheidungsfindungen massiv reduzieren. Dies sollte einen aber nicht davon abhalten, zu versuchen, die negativen Effekte so gut es geht zu reduzieren. Es gibt zwar Initiativen, die die Transparenz durch Maßnahmen wie ein verpflichtendes Lobbyregister ${ }^{5}$ erhöhen wollen, aber dies allein wird nicht ausreichen. In erster Linie muss daran angesetzt werden, dass Politik und Verwaltung ihre Entscheidungskompetenz nicht aus der Hand geben, egal wie verlockend und hilfreich manche Angebote erscheinen mögen. Dazu können auch Regelungen dienlich sein, die dann aber weit über ein Lobbyregister hinausgehen müssen. ${ }^{6}$ Von Kanzleien geschriebene Gesetzentwürfe, Interessenvertreter, die zeitweise mit enormem Einfluss in Ministerien arbeiten oder Wechsel von der Politik in die Wirtschaft stellen Herausforderungen für die Demokratie dar, die sich auch durch mehr Transparenz nicht lösen lassen.

Die Repräsentation von Interessen gegenüber politischen Entscheidungsträgern gehört fest zu einer Demokratie. Entscheidend ist, ob die tatsächliche Entscheidung am Ende von einer gewählten Person getroffen wird, oder nicht. Mut macht hierbei, dass Bernhagen (2012) in einer Studie zeigen konnte, dass es nur in wenigen Fällen gelingt, die Entscheidung von Politikern in einer Art und Weise zu drehen, die ihren eigentlichen politischen Zielen widerspricht. Dazu ist es allerdings wichtig, dass gerade politische Entscheidungsträger sehr genau verstehen, was Grenzen und Nutzen der Public Affairs für sie selbst, aber auch für die Demokratie sind.

\section{Literatur}

de|ge|pol - Deutsche Gesellschaft für Politikberatung e.V. (2010). Was ist Politikberatung? Berater, Auftraggeber, Tätigkeiten und die de|ge|pol als Berufsverband. https://static1.squarespace.com/ static/559675bfe4b0d2540b662c7c/t/559a9338e4b069786e95d592/1436193592653/was ist politikberatung.pdf. Zugegriffen am 09.12.2019.

de|ge|pol - Deutsche Gesellschaft für Politikberatung e.V. (2017). de'ge'pol fordert für gutes Lobbying mehr als ein Lobbyregister. https://www.degepol.de/aktuelles/degepol-fordert-fr-gu tes-lobbying-mehr-als-ein-lobbyregister08.02.2017. Zugegriffen am 10.12.2019.

Ainsworth, S. (1993). Regulating lobbyists and interest group influence. Journal of Politics, 55(1), $41-56$.

Bach, T., \& Wegrich, K. (2019). Das Verhältnis von Regierung und Verwaltung aus internationaler Perspektive. In A. Ritz, T. Haldemann \& F. Sager (Hrsg.), Blackbox Exekutive (S. 65-87). Basel: NZZ Libro.

Baumgartner, F. R., Berry, J. M., Hojnacki, M., Leech, B. L., \& Kimball, D. C. (2009). Lobbying and policy change - Who wins, who loses, and why. Chicago: University of Chicago Press.

Bernhagen, P. (2012). When do politicians listen to lobbyists (and who benefits when they do)? European Journal of Political Research, 52(1), 20-43.

\footnotetext{
${ }^{5}$ Interessanterweise eine Forderung, die zunehmend aus den Public Affairs selbst kommt. Sowohl von Verbänden wie BDI, VCI oder Nabu (Neuhaus 2019), aber auch von Beratungsagenturen (de'ge'pol 2017).

${ }^{6}$ Vorschläge finden sich beispielsweise bei Leif (2010) oder auch bei de'ge'pol (2017).
} 
Bernhagen, P. (2019). Lobbyismus in der Politikberatung. In S. Falk, M. Glaab, A. Römmele, H. Schober \& M. Thunert (Hrsg.), Handbuch Politikberatung (S. 249-261). Wiesbaden: Springer VS.

Biegelbauer, P., Grießler, E., \& Loeber, A. (2013). Politik, öffentliche Verwaltung und Wissen. In S. Kropp \& S. Kuhlmann (Hrsg.), der moderne staat-Zeitschrift für Public Policy, Recht und Management, Sonderheft 1/2013: Wissen und Expertise in Politik und Verwaltung (S. 223-239). Opladen/Toronto: Barbara Budrich.

Bogumil, J. (2002). Zum Verhältnis von Politik- und Verwaltungswissenschaft in Deutschland. polis Nr. 54/2002 Arbeitspapiere aus der Politikwissenschaft an der Fern-Universität Hagen, 1-30.

Bogumil, J. (2003). Die politische Führung öffentlicher Dienste - Möglichkeiten und Grenzen der Reorganisation. In R. Koch \& P. Conrad (Hrsg.), New Public Service - Öffentlicher Dienst als Motor der Staats- und Verwaltungsmodernisierung (S. 61-78). Wiesbaden: Springer VS.

Bogumil, J., \& Jann, W. (2009). Verwaltung und Verwaltungswissenschaft in Deutschland. Einführung in die Verwaltungswissenschaft (2. Aufl.). Wiesbaden: Springer VS.

Bogumil, J., Ebinger, F., \& Jochheim, L. (2012). Spitzenbeamte und ihr Verhalten bei politisch relevanten Entscheidungen. In D. Schimanke, H. Bull \& S. Veit (Hrsg.), Bürokratie im Irrgarten der Politik (S. 151-174). Baden-Baden: Nomos.

Burkhardt, O. (2008). Die Einbindung privater Unternehmensberater in staatliche Entscheidungsprozesse. Externe Politikberatung im Lichte des Verfassungsrechts. Berlin: LIT.

Buzogány, A., \& Kropp, S. (2013). Der Deutsche Bundestag im „Tal der Ahnungslosen“? Wissen und Expertise des Parlaments in europäisierten Fachpolitiken. In S. Kropp \& S. Kuhlmann (Hrsg.), der moderne staat - Zeitschrift für Public Policy, Recht und Management, Sonderheft 1/2013: Wissen und Expertise in Politik und Verwaltung (S. 161-182). Opladen/Toronto: Barbara Budrich.

Chahoud, A. (2010). Blicke hinter die Kulissen - Aus dem „Alltag“ eines Politikberaters. Aus Politik und Zeitgeschichte, 19(2010), 34-38.

Daumann, F. (1999). Interessenverbände im politischen Prozeß - Einflußnahme und Möglichkeiten der Begrenzung. ORDO - Jahrbuch für die Ordnung von Wirtschaft und Gesellschaft, 50(1), 171-206.

Deutscher Bundestag. (2019). Aktuelle Fassung der öffentlichen Liste über die Registrierung von Verbänden und deren Vertretern. https://www.bundestag.de/resource/blob/189476/6649c2695f4 bc5007732622aa39b012d/lobbylisteaktuell-data.pdf. Zugegriffen am 15.11.2019.

Enke, D., \& Franke, S. (2019). Stripped to the core - ein praxisorientierter Blick auf modern Public Affairs-Arbeit. In S. Falk, M. Glaab, A. Römmele, H. Schober \& M. Thunert (Hrsg.), Handbuch Politikberatung (S. 517-533). Wiesbaden: Springer VS.

Falk, S., \& Römmele, A. (2009). Der Markt für Politikberatung. Wiesbaden: Springer VS.

Falk, S., Römmele, A., Schober, H., \& Thunert, M. (2010). Politikberatungsforschung. In E. Jesse, M. Kneuer, I. Gerlach \& N. Werz (Hrsg.), Politikwissenschaft in Deutschland (S. 97-121). Baden-Baden: Nomos.

Goodwin, J., Jasper, J. M., \& Polletta, F. (2001). Why emotions matter. In J. Goodwin, J. M. Jasper \& F. Polletta (Hrsg.), Passionate politics: Emotions and social movements (S. 1-24). Chicago: The University of Chicago Press.

Graser, A. (2019). Politikberatung aus juristischer Sicht. In S. Falk, M. Glaab, A. Römmele, H. Schober \& M. Thunert (Hrsg.), Handbuch Politikberatung (S. 39-50). Wiesbaden: Springer VS.

Grohs, S. (2015). Die verwaltungswissenschaftliche Perspektive auf Demokratie. In H. Breitmeier (Hrsg.), Politikwissenschaft, Verwaltungswissenschaft, Soziologie: eine interdisziplinäre Einführung (S. 91-114). Hagen: Fernuniversität Hagen.

Grundwald, A. (2008). Technik und Politikberatung - Philosophische Perspektiven. Frankfurt a. M.: Suhrkamp.

Habermas, J. (1968). Verwissenschaftliche Politik und öffentliche Meinung. In J. Habermas (Hrsg.), Technik und Wissenschaft als „Ideologie“ (S. 120-145). Frankfurt a. M.: Suhrkamp.

Head, B. W. (2010). Reconsidering evidence-based policy - Key issues and challenges. Policy and Society, 29(5), 77-94. 
Hustedt, T., Veit, S., \& Fleischer, J. (2010). Wissen ist Macht? Wissenschaftliche Politikberatung. Aus Politik und Zeitgeschichte, 19, 15-21.

Jun, U. (2019). Spitzenakteure der Parteien. In S. Falk, M. Glaab, A. Römmele, H. Schober \& M. Thunert (Hrsg.), Handbuch Politikberatung (S. 221-231). Wiesbaden: Springer VS.

Kloepfer, M. (2011). Gesetzgebungsoutsourcing - Die Erstellung von Gesetzentwürfen durch Rechtsanwälte. NJW - Neue Juristische Wochenschrift, 64(3), 131-134.

Knoblauch, H. (2010). Wissenssoziologie. Konstanz: UVK Verlagsgesellschaft.

Korinek, R., \& Veit, S. (2013). Wissenschaftliche Politikberatung als Grenzarbeit - Ein Konzept zur Analyse institutionalisierter Beratungsformen in Politikfeldern. In S. Kropp \& S. Kuhlmann (Hrsg.), der moderne staat - Zeitschrift für Public Policy, Recht und Management, Sonderheft 1/2013: Wissen und Expertise in Politik und Verwaltung (S. 261-284). Opladen/Toronto: Barbara Budrich.

Kozica, A., Brandl, J., \& Kaiser, S. (2013). Verwaltungsberatung - Wie lernen öffentliche Verwaltungen aus externer Beratung? In S. Kropp \& S. Kuhlmann (Hrsg.), der moderne staat Zeitschrift für Public Policy, Recht und Management, Sonderheft 1/2013: Wissen und Expertise in Politik und Verwaltung (S. 305-324). Opladen/Toronto: Barbara Budrich.

Kropp, S., \& Kuhlmann, S. (2013). Wissen und Expertise in Politik und Verwaltung - eine einleitende Zusammenfassung. In S. Kropp \& S. Kuhlmann (Hrsg.), der moderne staat Zeitschrift für Public Policy, Recht und Management, Sonderheft 1/2013: Wissen und Expertise in Politik und Verwaltung (S. 7-19). Opladen/Toronto: Barbara Budrich.

Kusche, I. (2008). Politikberatung und die Herstellung von Entscheidungssicherheit im politischen System. Wiesbaden: Springer VS.

Ledermann, S. (2014). Evidenz und Expertise im vorparlamentarischen Gesetzgebungsprozess: Die Rolle von Verwaltung und externen Experten. Swiss Political Science Review, 20(3), 453-485.

Leif, T. (2010). Von der Symbiose zur Systemkrise. Aus Politik und Zeitgeschichte, 19(2010), 3-9.

Leif, T., \& Speth, R. (2006). Die fünte Gewalt - Lobbyismus in Deutschland. Wiesbaden: VS.

Lentsch, J. M., \& Weingart, P. (2009). Scientific advice to policy making in comparative perspective - Technocracy revisited - Introduction. In J. Lentsch \& P. Weingart (Hrsg.), Scientific advice to policy making - International comparison (S. 7-16). Opladen/Farmington Hills: Barbara Buderich.

Lehmbruch, G. (1987). Administrative Interessenvermittlung. In A. Windhoff-Héritier (Hrsg.), Verwaltung und ihre Umwelt: Festschrift für Thomas Ellwein (S. 11-43). Opladen: Westdetuscher.

Lipsky, M. (1980). Street-level Bureaucracy. dilemmas of the individual in public services. New York: Russel Sage Foundation.

Luhmann, N. (1989). Legitimation durch Verfahren. Frankfurt a. M.: Suhrkamp.

Mayntz, R. (2006). Die Organisation wissenschaftlicher Politikberatung in Deutschland. In Heidelberger Akademie der Wissenschaften (Hrsg.), Politikberatung in Deutschland (S. 115-122). Wiesbaden: Springer VS.

Neuhaus, C. (2019). Sie wollen ihr schlechtes Image loswerden - Lobbyisten fordern Lobbyregister. https://www.tagesspiegel.de/wirtschaft/lobbyisten-fordern-lobbyregister-sie-wollen-ihr -schlechtes-image-loswerden/24502766.html. Zugegriffen am 10.12.2019.

Nicholson-Crotty, S. (2005). Bureaucratic competition in the policy process. Policy Studies Journal, 33(1), 341-361.

Nullmeier, F. (2013). Wissenspolitologie und interpretative Politikanalyse. In S. Kropp \& S. Kuhlmann (Hrsg.), der moderne staat - Zeitschrift für Public Policy, Recht und Management, Sonderheft 1/2013: Wissen und Expertise in Politik und Verwaltung (S. 21-43). Opladen/ Toronto: Barbara Budrich.

Nullmeier, F., \& Rüb, F. W. (1993). Die Transformation der Sozialpolitik: Vom Sozialstaat zum Sicherungsstaat. Frankfurt a. M./New York: Campus.

Oltmanns, T., \& Seidenglanz, R. (2020). Auf der Suche nach Partnerschaft. politik \& kommunikation. https://www.politik-kommunikation.de/ressorts/artikel/auf-der-suche-nach-der-partner schaft-477164454/. Zugegriffen am 09.03.2020. 
Peters, G. B. (1987). Politicians and Bureaucrats in the politics of policy making. In J. Lane (Hrsg.), Bureaucracy and public choice (S. 255-282). London: Sage.

Pierre, J., \& Peters, B. (2017). The shirking bureaucrat: A theory in search of evidence? Policy \& Politics, 45(2), 157-172.

Raffel, T. (2006). Unternehmensberater in der Politikberatung - Eine empirische Untersuchung zu Aktivitäten, Gründen und Folgen. Wiesbaden: Deutscher Universitätsverlag.

Römmele, A., \& Schober, H. (2013). Pluralismus ohne Vielfalt? Anpassungsprozesse und Alleinstellungsmerkmale in der externen Politikberatung. In S. Kropp \& S. Kuhlmann (Hrsg.), der moderne staat - Zeitschrift für Public Policy, Recht und Management, Sonderheft 1/2013: Wissen und Expertise in Politik und Verwaltung (S. 241-259). Opladen/Toronto: Barbara Budrich.

Rüb, F. (2006). Wissenspolitologie. In J. Behnke, T. Gschwend, D. Schindler \& K. Schnapp (Hrsg.), Methoden der Politikwissenschaft. Baden-Baden: Nomos.

Rudzio, W. (2015). Das politische System der Bundesrepublik Deutschland. (9., Ak. u. erw. Aufl.) Wiesbaden: Springer VS.

Sabatier, P. (1998). The advocacy coalition framework: Revisions and relevance for Europe. Journal of European Public Policy, 5(1), 98-130.

Schedler, K. (2003). „... And Politics?“ Public management developments in the light oft wo rationalities. Public Management Review, 5(4), 533-550.

Schedler, K., \& Eicher, A. (2013). Das Verhältnis von Verwaltung und Politik. In A. Ladner, J. Chappelet, Y. Emery, P. Knoepfel, L. Mader, N. Soguel \& F. Varone (Hrsg.), Handbuch der öffentlichen Verwaltung in der Schweiz (S. 369-384). Zürich: NZZ Libro.

Schedler, K., \& Proeller, I. (2000). New public management. Bern: Paul Haupt.

Schmedes, H. (2010). Das Mosaik der Interessenvermittlung im Mehrebenensystem Europas. Aus Politik und Zeitgeschichte, 19/2010, 22-27.

Schörner, M. (2017). Public Affairs, Government Relations und Lobbying. Die Interessenvertretung deutscher Großunternehmen gegenüber der Politik und Verwaltung in Deutschland. Münster: LIT.

Siefken, S. (2010). Ist denn alles Politikberatung? Anmerkungen zum Begriff und der Diagnose insitutionalisierter Kooperation. Politische Vierteljahresschrift, 1(2010), 127-136.

Simon, H. A. (1959). Theories of decision-making in economics and behavioral. Science. The American Economics Review, 49(3), 253-283.

Speth, R. (2004). Advokatorische think tanks. Bonn: Friedrich-Ebert-Stiftung.

Stehr, N. (1994). Knowledge societies. London: Sage.

Stöbe-Blossey, S. (2004). Beratung und Verwaltungsreform - Vom Gutachten zur aktiven Moderation. In D. Rehfeld (Hrsg.), Arbeiten an der Quadratur des Kreises - Erfahrungen an der Schnittstelle zwischen Wissenschaft und Praxis (S. 91-112). München/Mehring: Hampp.

Straßheim, H. (2013). Politische Expertise im Wandel. Zur diskursiven und institutionellen Einbettung epistemischer Autorität. In S. Kropp \& S. Kuhlmann (Hrsg.), der moderne staat Zeitschrift für Public Policy, Recht und Management, Sonderheft 1/2013: Wissen und Expertise in Politik und Verwaltung (S. 65-86). Opladen/Toronto: Barbara Budrich.

Van Schendelen, R. (2014). Politische Parteien und Interessengruppen auf der nationalen Ebene und in der EU: umgekehrte demokratische Verhältnisse? Zeitschrift für Parlamentsfragen, 3, 669-692.

Von Alemann, U., \& Eckert, F. (2006). Lobbyismus als Schattenpolitik. Aus Politik und Zeitgeschichte, 2006, 15-16, 3-10.

Vorderstraße, S. (2014). Zeit und Politikberatung. Wiesbaden: Springer VS.

Wagschal, U. (2019). Politikberatung und demokratische Legitimität. In S. Falk, M. Glaab, A. Römmele, H. Schober \& M. Thunert (Hrsg.), Handbuch Politikberatung (S. 51-66). Wiesbaden: Springer VS.

Weber, M. (1985). Wirtschaft und Gesellschaft. Tübingen: J.C.B. Mohr.

Weingart, P. (2019). Wissensgesellschaft und wissenschaftliche Politikberatung. In S. Falk, M. Glaab, A. Römmele, H. Schober \& M. Thunert (Hrsg.), Handbuch Politikberatung (S. 67-78). Wiesbaden: Springer VS. 
Weingart, P., \& Lentsch, J. M. (2008). Wissen - Beraten - Entscheiden - Form und Funktion wissenschaftlicher Politikberatung in Deutschland. Weilerswist: Velbrück Wissenschaft.

Widmer, T. (2009). The contribution of evidence-based policy to the output-oriented legitimacy of the state. Evidence \& Policy, 5(4), 251-372.

Wonka, A. (2005). Lobbying des Europäischen Parlaments. In A. Maurer \& D. Nickel (Hrsg.), Das Europäische Parlament. Supranationalität, Repräsentation und Legitimation (S. 165-172). Baden-Baden: Nomos. 